IOSR Journal of Engineering (IOSRJEN)

e-ISSN: 2250-3021, p-ISSN: 2278-8719

Vol. 3, Issue 4 (April. 2013), ||V1 || PP 43-48

\title{
Face Databases for 2D and 3D Facial Recognition: A Survey
}

\author{
R.Senthilkumar ${ }^{1}$, Dr.R.K.Gnanamurthy ${ }^{2}$ \\ ${ }^{1}$ Assistant Professor, Department of Electronics and Communication Engineering, Institute of Road and \\ Transport Technology,Erode-638 316. \\ ${ }^{2}$ Professor and Dean, Department of Electronics and Communication Engineering, Odaiyappa College of \\ Engineering and Technology,Theni-625 531.
}

\begin{abstract}
This paper presents a detailed case study of different face databases frequently used for face recognition and object recognition. The simple face database developed at AT \& T lab contains 40 subjects each with 10 images. The database was collected by AT \& T lab and it has the subjects of different pose and facial expressions. The yale database contains 15 subjects each with 11 images. The facial recognition technology database contain more than 4,000 both gray scale and colour images. Different 2D face recognition algorithms used this database for their performance comparison. The cmu pie and multi-pie databases were developed for testing the performance of different video and still face recognition algorithms. The photometric stereo (PS) is a method to rapidly estimate the three-dimensional geometry of a face (or any other Lambertian-like object) using several images with an identical viewpoint but varied illumination directions. This paper work help to select appropriate face databases for both video face and still face recognition and also it gives an idea about selection of databases for 2D and 3D face recognition algorithms.
\end{abstract}

Keywords: Face database, face recognition, feret, labeled faces, multi-pie, photometric stereo, yale database.

\section{INTRODUCTION}

There have been a plethora of face recognition algorithms and related face databases published in the literature during the last few decades. During this time, a great many databases of face images, and later 3-D scans, have been collected as a means to test these algorithms. Section II describes about the ORL (now simple face database) database [8],[11]. All the images of the subjects are 2D images. Section III describes about the yale database [12],[13]. The images in this database are suitable for 2D still image face recognition methods. The ORL and yale database both contain gray scale face images. The Facial Recognition Technology (FERET) Database [4],[9] contains both gray scale and colour images and it is discussed briefly in section IV. The pie, multi-pie are discussed in section V and VI respectively. Section VII explains the usage of labeled in the wild database for webpage based facial recognition. The application of PS device in face recognition is explained in section VIII. Section IX covers the concluding part.

\section{ORL DATABASE}

Our Database of Faces, (formerly 'The ORL Database of Faces'), contains a set of face images were taken between April 1992 and April 1994 at the AT\&T lab. The database was used in the context of a face recognition project carried out in collaboration with the Speech, Vision and Robotics Group of the Cambridge University Engineering Department. There are ten different images of each of 40 distinct subjects [8],[11]. For some subjects, the images were taken at different times, varying the lighting, facial expressions (open / closed eyes, smiling / not smiling) and facial details (glasses / no glasses). All the images were taken against a dark homogeneous background with the subjects in an upright, frontal position (with tolerance for some side movement). A preview image of the Database of Faces is available. The files are in PGM format, and can conveniently be viewed on UNIX (TM) systems using the 'xv' program. The size of each image is $92 \times 112$ pixels, with 256 gray levels per pixel as shown in Fig.1. The images are organized in 40 directories (one for each subject), which have names of the form $s X$, where $X$ indicates the subject number (between 1 and 40). In each of these directories, there are ten different images of that subject, which have names of the form Y.pgm, where $\mathrm{Y}$ is the image number for that subject (between 1 and 10).
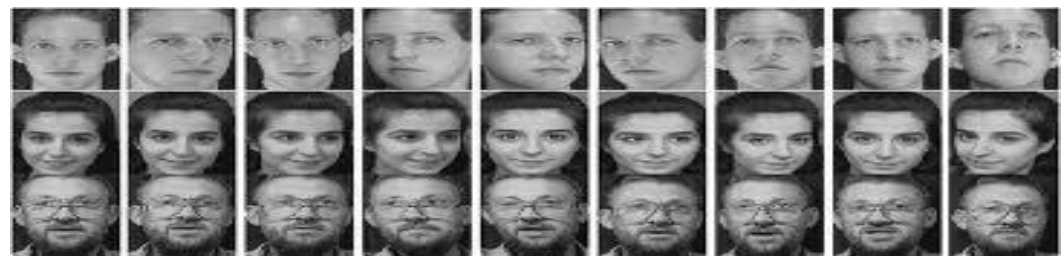

Fig.1. Three indivuals of ORL database each with 10 images (Nine images are shown). 


\section{YALE FACE DATABASE}

The Yale Face Database (size 6.4MB) [12],[13] contains 165 grayscale images in GIF format of 15 individuals. There are 11 images per subject as shown in Fig.2, one per different facial expression or configuration: center-light, with glasses, happy, left-light, without glasses, normal, right-light, sad, sleepy, surprised, and wink.

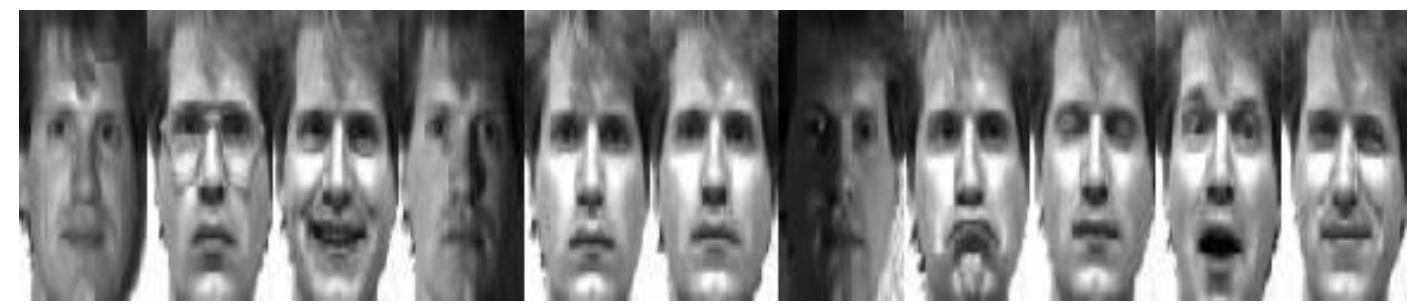

Fig.2. A sample of 11 images of single subject.

\section{FACIAL RECOGNITION TECHNOLOGY DATABASE}

The FERET program ran from 1993 through 1997, sponsored by the Department of Defense's Counterdrug Technology Development Program through the Defense Advanced Research Products Agency (DARPA). Its primary mission was to develop automatic face recognition capabilities that could be employed to assist security, intelligence and law enforcement personnel in the performance of their duties.

The FERET image [4], [9] corpus was assembled to support government monitored testing and evaluation of face recognition algorithms using standardized tests and procedures. The final corpus consists of 14051 eight-bit grayscale images of human heads with views ranging from frontal to left and right profiles.
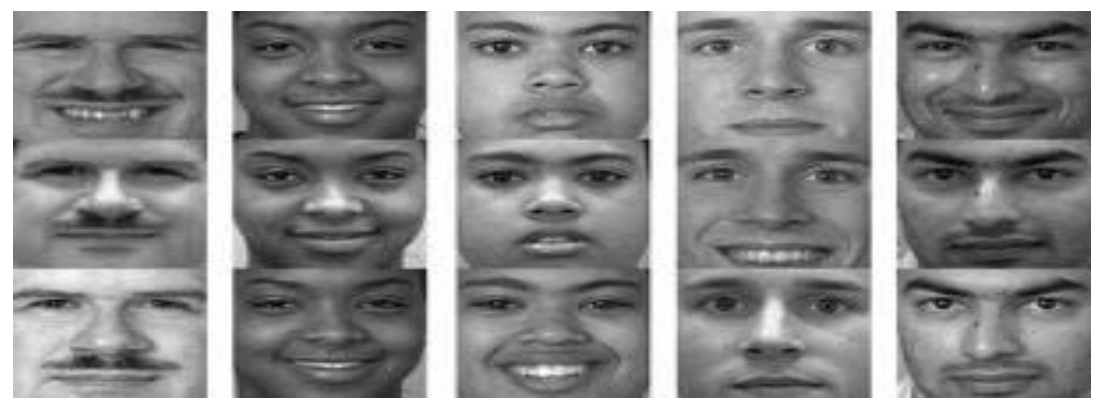

Fig.3. Samples of cropped images of 5 subjects with each 3 images.

\section{CMU PIE (POSE, ILLUMINATION AND EXPRESSION) DATABASE}

It consists of a face database of 41,368 images of 68 people [10]. By extending the CMU 3D Room, images of each person under 13 different poses, 43 different illumination conditions, and with 4 different expressions were captured.

The CMU PIE database [10] has been very influential in advancing research in face recognition across pose and illumination. Despite its success the PIE database has several shortcomings: a limited number of subjects, single recording session and only few expressions captured.

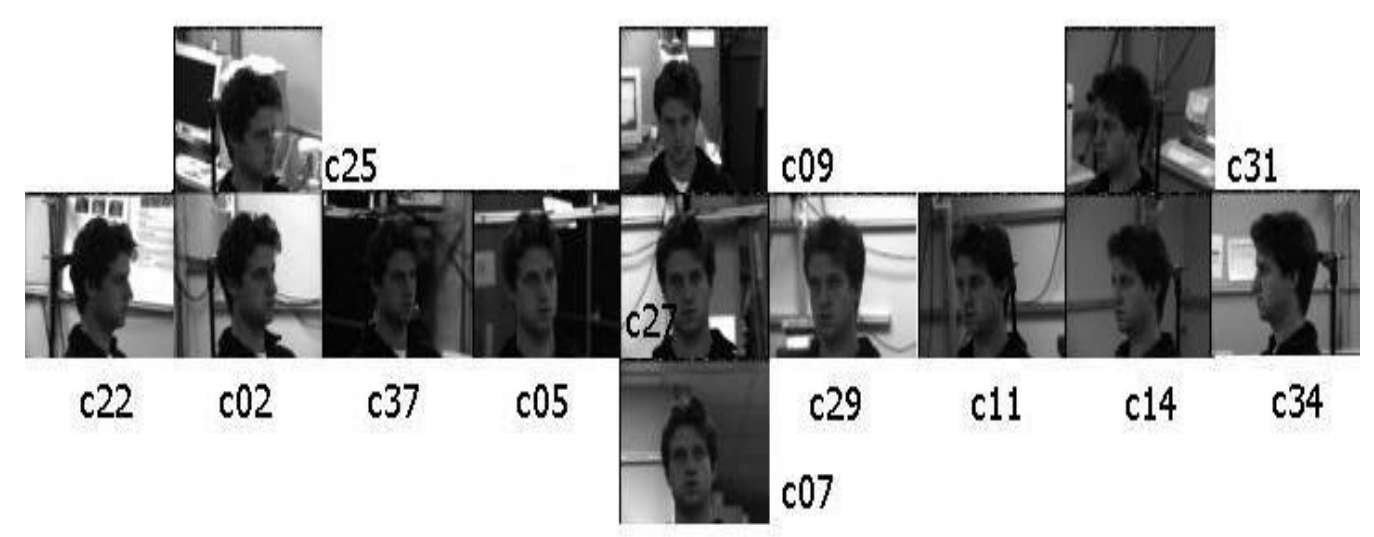

Fig.4. Sample Images of a sing le person in CMU PIE database. 


\section{CMU MULTI-PIE DATABASE}

It contains 337 subjects, imaged under 15 view points and 19 illumination conditions in up to four recording sessions [2],[3]. To systematically capture images with varying poses and illuminations, a system of 15 cameras and 18 flashes connected to a set of Linux PCs were used. Thirteen cameras were located at head height, spaced at $15 \hat{\mathrm{A}}^{\circ}$ intervals, and two additional cameras were located above the subject, simulating a typical surveillance view. The following Fig.5 illustrates the location of the cameras.

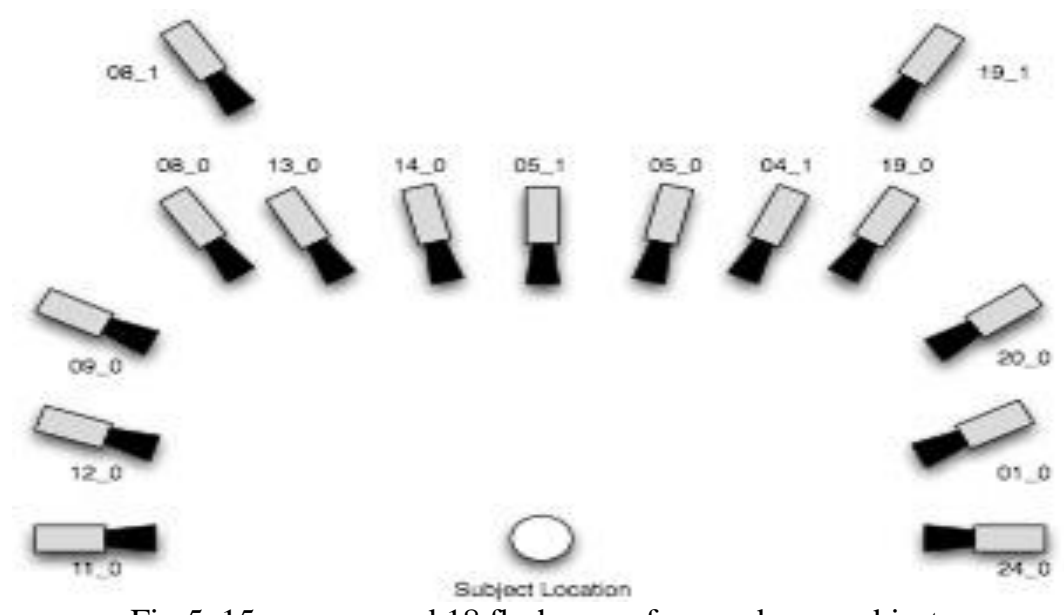

Fig.5. 15 cameras and 18 flashes are focussed on a subject.

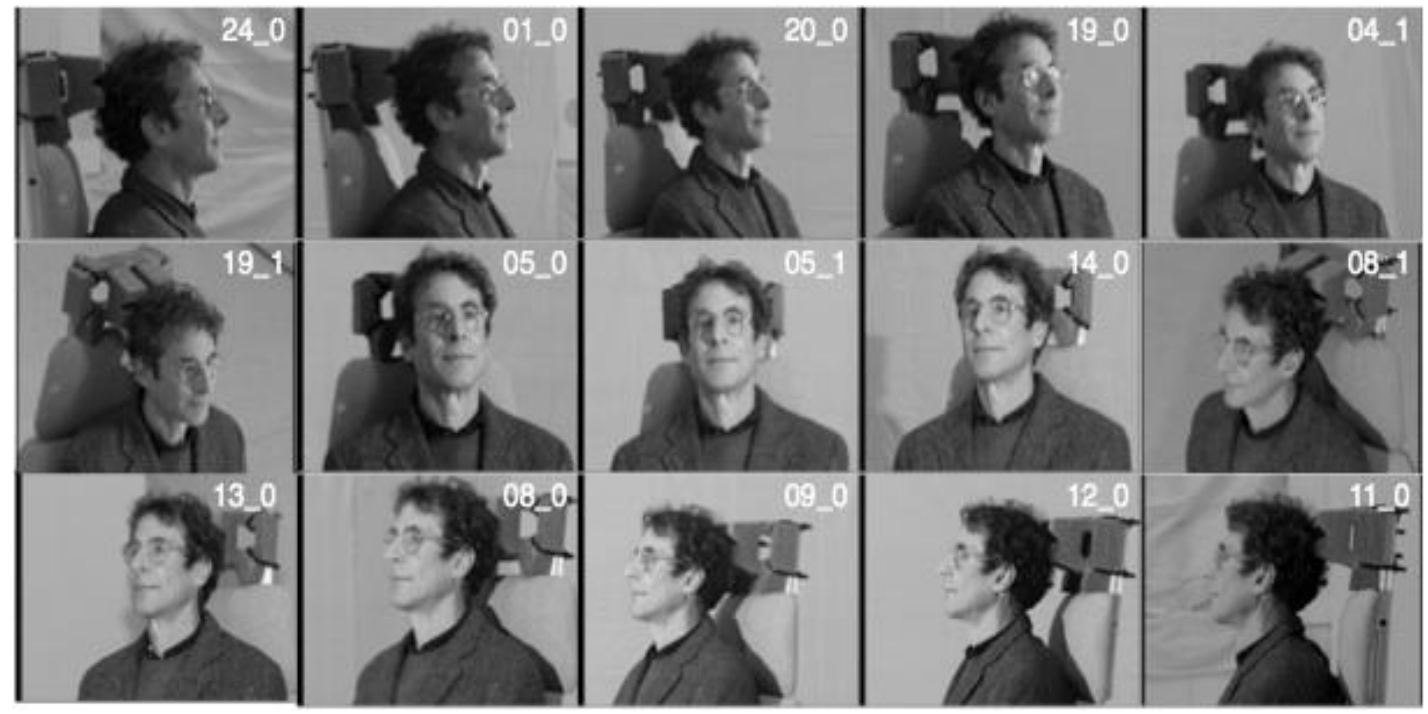

Fig.6. Shows all 15 camera views with frontal flash illumination.

The frontal images were recorded using a Canon EOS 10D (6.3-megapixel CMOS camera) with a Macro Ring Lite MR-14X ring flash. Subjects were seated in front of a blue background in close proximity of the camera. The resulting images are 3072 x 2048 in size with the inter-pupil distance of the subjects typically exceeding 400 pixels.

\section{LABELED FACES IN THE WILD (LFW) DATABASE}

The Labeled Faces in the Wild (LFW) images and its benchmark [5], reflect the challenges of face recognition from unconstrained images. Recognizing faces appearing in unconstrained, natural conditions remains a challenging task. The LFW dataset in particular is published with a specific benchmark, which focuses on the face recognition task of pair matching. In this task given two face images, the goal is to decide whether the two pictures are of the same individual.

The LFW database as shown in Fig.7 offers a unique collection of around 13,000 annotated faces automatically captured from news articles on the web. The images in this set are partitioned into 5,749 individuals each having from one to 150 images. These images are paired into 6,000 pairs of images, half labeled "same" and half "not same". These pairs are further divided between ten test splits mutually exclusive in the subject identities (a person appearing in one will not appear in the other nine). 


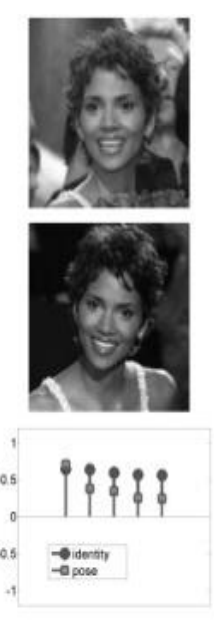

(a)
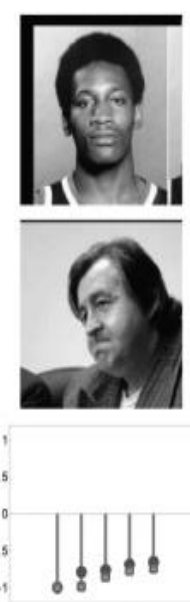

(b)
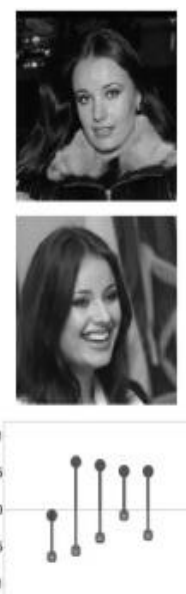

(c)
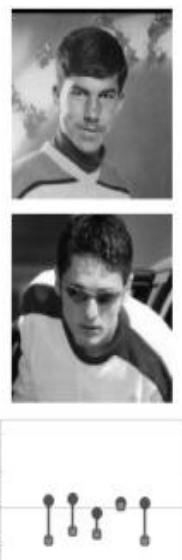

(d)

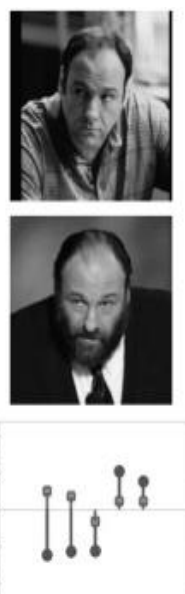

(e)

Fig.7. Each group contains two images and 10 sample multiple

OSS scores. Identity based multiple OSS scores are plotted with circle markers and pose based are with squares. As can be seen the value of each type of OSS score is a good indication of the type of similarity between the images of the pair. (a) Same person, same pose. (b) Different persons and pose. (c) Same person, different pose. (d) Different persons, same pose. (e) Same person and pose, however, a mode of variability not modeled in the system is present. One of the nine training splits for the background set ' $\mathrm{A}$ ' and the other eight for classifier training. The background split contains 1,200 images. Multi-identity face classification in the LFW data set, two images per person as probes and two as gallery, a subset of LFW image set consisting of the 610 subjects having at least four images have been taken. This subset contains a total of 6733 images.

The best results currently reported on the LFW benchmark were obtained by using the one shot similarity measure. But "Two-shot similarity"(TSS)[1], score as an extension to the recently proposed "one-shot similarity" (OSS) measure, shows better performance than OSS. Both these measures utilize background samples to facilitate better recognition rates.

\section{PHOTOMETRIC STEREO DATABASE}

The photometric stereo database [6], suitable for both 2D and 3D face recognition. The database was collected using a custom-made four-source PS device designed to enable data capture with minimal interaction necessary from the subjects. The device, which automatically detects the presence of a subject using ultrasound, was placed at the entrance of a busy workplace and captured 1839 sessions of face images with natural pose and expression. This meant that the acquired data is more realistic for everyday use than existing databases and is therefore an invaluable test bed for stat-of-the-art recognition algorithms.

The photoface database was selected using the custom-made four-source PS device shown in Fig.8. The presence of an individual is detected by an ultrasound proximity sensor placed before the archway.

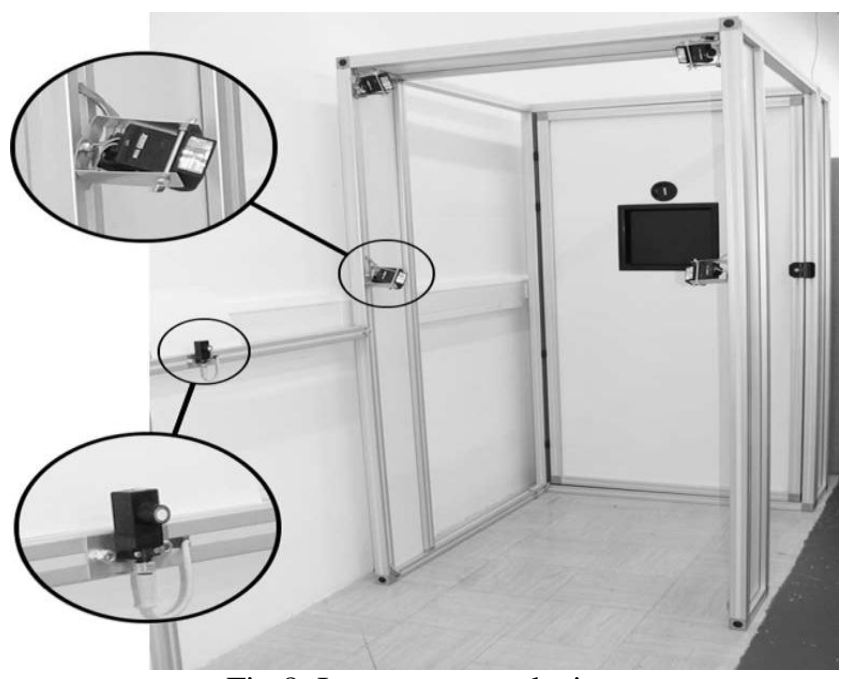

Fig.8. Image capture device. 
One of the light sources and an ultrasound trigger are shown to the left. The camera is located at the back panel. The device also contains a monitor that provides instructions and could be used to indicate whether or not an individual was recognized in the case of a recognition scenario, or whether an identity claim was accepted or rejected in the case of a verification scenario. The Fig.9. shows an example of four raw images of an individual. The resolution of the original image captured is $1280 \times 1024 p x$, although the images in our database automatically to the face itself typically of the order $600 \mathrm{x} 800 \mathrm{px}$.

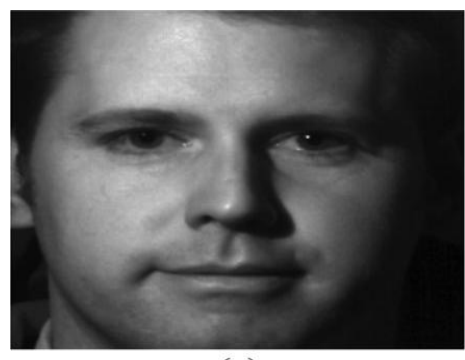

(a)

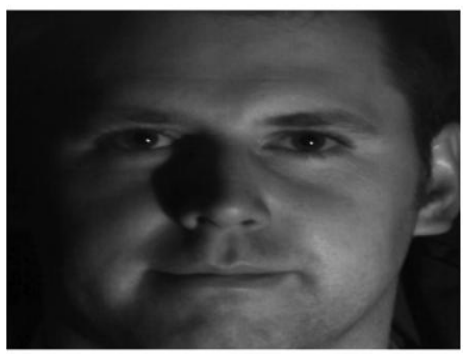

(c)

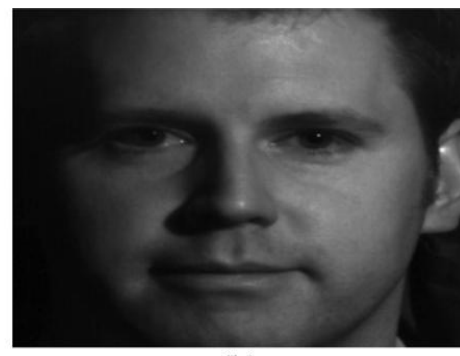

(b)

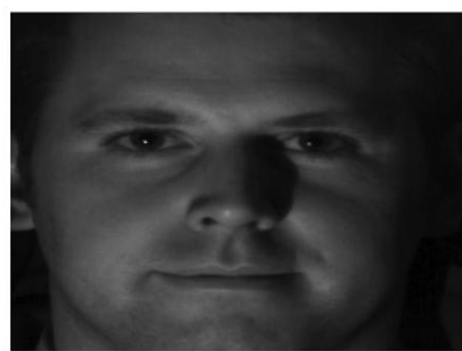

(d)

Fig.9. Four images taken under the different lights (only the facial region).

The photoface database consists of a total of 1839 sessions of 261 subjects and a total of 7,356 images. The majority of people in the database are male 227 and 34 female. Regarding repeat usages, 98 people walked through the device only once. For 126 of the 163 subjects that used the device more than once, the sessions were collected over a period of more than a week's interval. The number of images corresponding to the number of subject recordings by the device is depicted in Fig. 10.

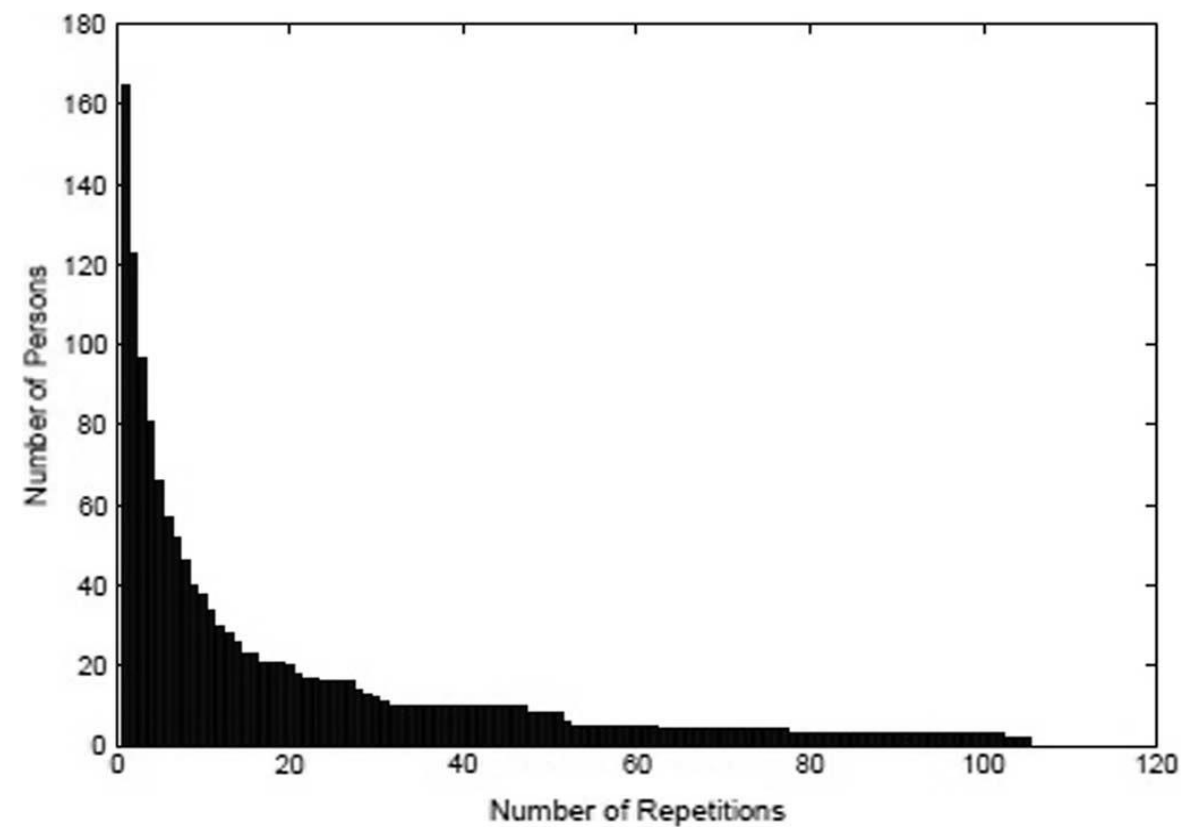

Fig.10. Number of subjects versus number of repetitions.

The PS inherently allows any combination of 2D data, 3D data or surface normal [7] to be used for recognition. The PS device offers many advantages over other face-shape capture devices in that it is competitive in terms of cost, computational efficiency, resolution capabilities and ease of calibration. 


\section{CONCLUSION}

In this paper work, some important 2D and 3D face databases frequently used for face recognition were covered. The ORL database is restricted to different pose and facial expressions. It contains 40 samples only. The yale database have subjects of different pose and facial expressions along with different illumination conditions. Both yale and ORL databases are restricted to few subjects only. The FERET gray scale and colour image face database provides sufficient subjects and the purpose of the tests was to measure overall progress in face recognition, determine the maturity of face recognition algorithms, and have an independent means of comparing algorithms. The tests measure the ability of the algorithms to handle large databases, changes in people's appearance over time, variations in illumination, scale, and pose, and changes in the background. The algorithms tested are fully automatic, and the images presented to the algorithm are not normalized. If an algorithm requires that a face be in a particular position, then the algorithm must locate the face in the image and transform the face into the required predetermined position.

The PIE database has several shortcomings: a limited number of subjects, single recording session and only few expressions captured. To address these issues the CMU Multi-PIE database was developed. It contains 337 subjects, imaged under 15 view points and 19 illumination conditions in up to four recording sessions. The LFW database along with its benchmark used to compare the performance of a large variety of existing video and still image face recognition techniques. The photometric stereo database suitable for both 2D and 3D face recognition. The four source PS methods produce facial samples (albedo, normal) that achieve constantly better recognition and verification performance than any other databases.

\section{REFERENCES}

[1]. L. Wolf, T. Hassner, and Y. Taigman,Similarity scores based on background samples, in Proc. Asian Conf. Comp. Vis. (ACCV), Sept. 2009.

[2]. R.Gross, I.Matthews, J.Cohn, T.Kanade, \& S.Baker, CMU multi-pie Face Database, in Proc.Intl.Conf. Aut. Face Gest. Recg, vol. 28, no.5, pp: 807-813, May.2010.

[3]. R.Gross, I.Matthews, J.Cohn, T.Kanade, \& S.Baker, Multi-pie, Image Vis. Comput., vol.28, pp.807$813,2010$.

[4]. P. J. Phillips, H. Moon, P. J. Rauss, and S. Rizvi, The FERET evaluation methodology for face recognition algorithms, IEEE Transactions on Pattern Analysis and Machine Intelligence, Vol. 22, No. 10, October 2000.

[5]. L.Wolf, T.Hassner, and Y.Taigman, Effective unconstrained face recognition by combining multiple descriptors and learned background statistics, IEEE Trans. Pattern Anal. Mach. Intell., vol.33,no.10,pp. 116, Oct.2011.

[6]. S.Zaefanos, G .A.Atkinson, M.F.Hansen, and William.A.P.Smith,Face recognition and verification using photometric stereo:The photoface database and a comprehensive evaluation., IEEE Trans. Inf. Forensics Security, vol.8, no.1, Jan.2013.

[7]. G.A.Atkinson, and M.L.Smith, Using photometric stereo for face recognition, Intl..J.Bio-Sci. and BioTech., vol.3, no.3, pp.35-44, Sep.2011.

[8]. M.Turk and A.P.Pentland, Eigenfaces for recognition, J.Cognitive Neurosci., vol.3, no.1, pp.71-86, 1991.

[9]. P.J.Phillips,H.Weshcler, P.Rauss, The ferret database and evaluation procedure for face-recognition algorithms, Image Vis. Comput., vol.16, pp.295-306, 1998.

[10]. T.Sim, S.Baker, and M.Bsat, The CMU pose, illumination, and expression database, IEEE Trans. Pattern Anal. Mach. Intell., vol.25, no.12, pp.1615-1618, Dec. 2003.

[11]. http://www.cl.cam.ac.uk/research/dtg/attarchive/facedatabase.html.

[12]. http://cvc.yale.edu/projects/yalefaces/yalefaces.html.

[13]. http://vismod.media.mit.edu/vismod/classes/mas622-00/datasets/YALE. 